been passed for construction by our own Nuclear Installations Inspectorate (NII). Indeed much of the book, if written in a more convincing tone, would form a very powerful argument for the establishment in the US of just such an independent body as our NII which would have to accept and pass was permitted. It may be that the lack of success of British industry in selling reactors abroad is due, not to the inefficiency often imputed, but to the fact that the installation of desirable safeguards has made them so much more expensive than American rethe full designs before construction

actors without such safeguards.

To summarise, quite a lot of the book is useful so long as one reads it not as a guide to the nuclear energy controversy, which it certainly is not, but as a highlighting of the risks, from the small but probable to the enormous but highly improbable, and as a very revealing statement of the point of view of the opponents of nuclear power.

J. H. Fremlin is Professor of Applied Radioactivity at the University of Birmingham, and was Technical Consultant to the Cumbria County Council at the Windscale Inquiry.

\section{Supernovae astronomy}

The New Astronomy. By P. Murdin and L. Murdin. Pp. 215. (Reference International: London; Thomas Y. Crowell: New York, 1978.) £5.95; $\$ 12.95$.

Anyone who has lectured to an amateur astronomical society, or a school class, knows that it is the question from the youngest member of the audience, from the least expert of the assembled, that is usually the most difficult to answer. I'll never forget my very first lecture, which revealed me as the green-horn $I$ was. After expounding on the glories of solar physics the first questioner, a seven year old, simply asked me "Why was the Sun called Sun?". Exit lecturer in confusion.

In general it is the hard, new, incomplete and speculative branches of astronomy that interest the lay students. Subjects that are well known and explicable are dismissed as 'boring'. Just mention blackholes, supernovae, curved space, pulsars and quasars and you can literally see the ears prick up and the whiskers twitch.

The Murdins' book is for these people. It reads like a good novel; it is hard to put down and can be gobbled up in one go. The hero of the piece is the supernova of $\mathrm{AD} 1054$ and its present day association, the Crab Nebula, Messier 1 in Taurus. As such the title of the book is a touch misleading. It should really be Supernovae Astronomy.

Paul and Lesley Murdin take to their hearts Geoffrey Burbidge's aphorism "contemporary astronomy can be divided into the study of the Crab Nebula and the study of everything else". An exaggeration obviously, but supernovae, their gas remnants, pulsars. blackholes, radio, $\mathrm{X}$-ray and neutrino emissions and their roles as cosmic ray sources, birth places of the heavy elements and possible triggers for planetary formation, do hold a central position in modern astronomy.

The mysteries of supernovae are revealed chronologically, the authors starting with the Chinese descriptions on 4 July, 1054. The Renaissance supernovae of 1574 and 1604 are discussed in detail, special emphasis being made of the way in which these two new stars shattered the crystal spheres of Aristotelean thought. In the old astronomy celestial perfection started beyond the Moon and the firmament of stars was immutable. This was disproved by Tycho Brahe's measurements of the parallax of his nova, a measurement which placed it at least ten times further away than the Moon.

\section{Paper and thin-layer chromatography}

Laboratory Handbook of Paper and Thin-Layer Chromatography. By J. Gasparic and J. Churacek. Pp. 362. (Wiley: New York and Chichester, 1978). $£ 18$

THF blurb on the dustcover of this book is most revealing. First. it tells us that "exhaustive references include the latest experience and results from East European sources.". Yet $95^{\prime \prime \prime}$ of the references are pre-1974 and 90\% pre1970 , which will fill many of us with envy at the thought of the very long holidays they must have. Second, it tells us that the two authors have already written "five books on paper and thin layer chromatography' and one can well believe it from a viewing of this, their sixth, work.

Part 1 comprises seventyseven pages and describes the principles, techniques and uses adequately. Part 2, the rest of the book, is devoted to the applications of the guest star which was first noted
The fact that the Galaxy should have a bright supernova every 200 years but that none has occurred since the invention of the telescope, is bemoaned. Hartwig's discovery of SN Andromedae in 1885 and its role in the Great Debate is mentioned. Then follows an account of how the veils of mystery of the Crab Nebula were peeled off one by one: Winlock and Pickering (1868) found that it was gaseous; Slipher (1913) found that it was expanding; Hubble (1928) connected it with the supernova of 1054; Baade (1945) noticed that the expansion was accelerating; Bolton and Stanley (1948) identified the radio star Taurus A as the Crab Nebula; X-ray astronomers at the Naval Research I aboratory. Washington (1963), discovered the Crab to be an X-ray source; Hewish and Bell (1968) discovered pulsars; and Staelin and Reifenstein searched the Crab and found one there; and Cooke. Disney and Taylor found light flashes emanating from the centre. It is a long list and one which will surely continue. The book ends with chapters on neutron stars, neutrino astronomy, element creation, cosmic rays and blackholes.

The New Astronomy by Paul and Lesley Murdin is a feast for the amateur astronomer and can be highly recommended.

David W. Hughes

David Hughes is Lecturer in Physics and Astronomy at the University of Sheffield, $U K$.

which cover the whole range of inorganic, organic and biological chemicals. However. about half of this section is devoted to tables of $R_{t}$ values, solvent and reagent compositions drawn entirely from the literature in what seems to be a fairly uncritical manner. A more careful look at the sections which particularly interest me shows that a total of two pages are devoted to amino acids. one to ketoacids, five to sugars and seven to lipids, and the whole subject of radioactive compounds is covered in less than three pages.

The professional analyst will certainly know far more about the subject than what is included in this work. whereas the student will need a much more critical and explanatory book. At all levels, there are better and far less expensive books available which I would recommend in preference to this $£ 18$ text

Ivor Smith

Ivor Smith is Reader in Biochemical Education at the Courtauld Institute. Middlesex Hospital Medical School, London, UK. 